\title{
Localization of Cooperative WSN using Distributed PSO with Optimum References
}

\author{
Ravichander Janapati ${ }^{1}$, Ch. Balaswamy ${ }^{2}$, K. Soundararajan ${ }^{3}$ \\ ${ }^{1}$ Department of ECE, SR Engineering College, Warangal, India \\ ${ }^{2}$ Department of ECE, QIS Engineering College Ongole, India \\ ${ }^{3}$ Department of ECE, TKR Engineerig College, Hyderabad, India
}

\begin{tabular}{|c|c|}
\hline Article Info & ABSTRACT \\
\hline & \multirow{5}{*}{$\begin{array}{l}\text { In indoor environment WSN nodes are deployed randomly and do not know } \\
\text { the accurate position. Find the node position with the help of anchor nodes is } \\
\text { known as localization. CRB algorithm selects the best anchor nodes which } \\
\text { gives high accuracy. In this paper distributed PSO algorithm with optimum } \\
\text { selection of reference nodes using CRB is proposed to find accurate node } \\
\text { position. The proposed method performs better in comparison with other } \\
\text { algorithms like PSO, RLS, LMS and GPS in terms of position accuracy, } \\
\text { latency and complexity. }\end{array}$} \\
\hline Received Apr 3, 2016 & \\
\hline Revised Jul 17, 2016 & \\
\hline Accepted Aug 1, 2016 & \\
\hline Keyword: & \\
\hline
\end{tabular}

Crammer rao bound (CRB)

Distributed localization

Distributed PSO

Particle swarm optimization

(PSO)

Copyright $\odot 2016$ Institute of Advanced Engineering and Science. All rights reserved.

Corresponding Author:

Ravichander Janapati,

Department of ECE,

SR Engineering College,

Warangal, India.

Email: ravi_chander_j@ srecwarangal.ac.in

\section{INTRODUCTION}

WSN is a collection of autonomus, distributed devices which are cooperativey send their information through the network to the central node. WSN can be used in critical applications of target tracking, health and military applications etc. [1]. In indoor environment nodes are deployed randomly and do not know their position. Finding exact node position is known as localization. GPS can be used to find node locations,due to noise and multipath effects it does not works well in indoor environment [2].

Localization algorithms can be used to find location of nodes using anchor nodes. Localization algorithms can be classified into two types: 1) Centralized localization algorithm 2) Distributed localization algorithm. In centralized localization algorithm central node calculates node location and passed to other nodes. Disadvantage of this method is complexity is more.Centralized algorithms are generally not scalable so unfeasible for large networks. In distributed localization algorithms each node computes its location hence complexity is reduced. Distributed algorithms are scalable so fit for large networks [3].

Nodes with known position is termed as anchor nodes, nodes do not know their position is known as agent nodes. In this localization process anchor nodes broadcast beacon signal and estimate location of un known nodes using RSS. All the anchor nodes does not estimate the location of nodes with high accuracy. Hence CRB is used to select the best reference nodes with high accuracy. Distributed PSO localization algorithm is proposed to find accurate node location. Proposed solution perform better in comparison with other algorithms like PSO, RLS algorithm, LMS algorithm and GPS in terms of position accuracy, latency and complexity. 
Organization of the paper is as follows, Section 2 gives problem statement, proposed Cooperative Distributed PSO with optimum selection of references using CRB is given in Section 3, simulation results \& analysis are given in Section 4, Section 5 gives conclusion.

\section{PROBLEM STATEMENT}

In non cooperative location estimation anchor nodes find location of agent node. Agent nodes do not participate in localization process because of communication does not exist between agent nodes. Main disadvantage of this method is that more anchor nodes or long distance transmissions are required. Cooperative localization method get the location information from anchor nodes and agent nodes, hence long range transmissions are not required [4].

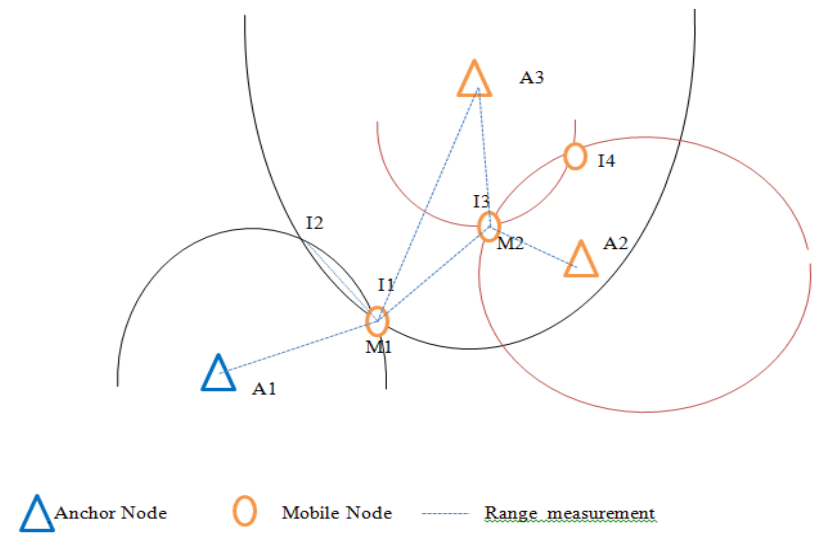

Figure 1. Cooperative network

To solve the problem of difficulties of a low number of reliable anchor nodes it might be helpful to get the mobile nodes cooperate for localization. Figure 1 shows the manner in which two mobile nodes get advantage from cooperation. Mobile node M1 is connected with anchor node A1 and A3 and mobile node M2 is connected with anchor nodes A2 and A3. Generally in range based localization both mobile node M1 and M2 cannot openly fix their locations. Mobile node M1 might be positioned in either interSection of I1 or I 2 whereas mobile node M2 might be in I3 or I4. In a cooperative WSN, nodes cooperated with each other and find the distance between them. This distance information is useful to find location of M1 is in I1 and M2 in I3. Cooperative localization needs communication between the mobile nodes to assist them to aid each other. In the example of Figure 1 that could be mobile node M1 sending its possible position coordinates of I1 and 2 to mobile node M2 that would then be able to determine its position unambiguously using the inter distance of the two mobile nodes. The position of M2 should then be returned to M1 assisting it to as well determine its position. Cooperative localization can compromise augmented accuracy and coverage.

The performance of positioning accuracy can be extremely enhanced by exchanging positional information between agents. Cooperative localization method improves positioning accuracy [5], but increases the computational complexity, network traffic and delay. Some of the positional information may also be destructive as some devices have poor estimates [6]. Hence, a big challenge is to selection and rejection of information from nodes. In the [7] proposed to use the links which are the closest from the agent in consideration. However, the closest neighbors may not correspond to the best links as positioning also depend on the geometric configuration of the agent and its neighbors. So selecting the anchor nodes to determine position of node is a big task. The Cramer Rao bound (CRB) used to discard vain links [8]. Rather than depend on proximity as a criterion, [9] proposed to use a preset number of links that give lowest CRB. In [10-11] the authors proposed to use the CRB to select those anchors that would give the best positioning accuracy. Distributed PSO localization algorithm is proposed to find accurate node location.

\section{COOPERATIVE DISTRIBUTED PSO LOCALIZATION USING OPTIMUM REFERENCES}

Proposed solution consists of two parts 1. Selection of optimum references 2. Distributed PSO localization. Section 3.1 explains optimum selection of references, Section 3.2 explains CDPSO with CRB localization algorithm. 


\subsection{Optimum Selection of References using CRB}

In cooperative WSN every node communicated with all other nodes. Every node involved in the process of estimation. Here CRLB is calculated to estimate the set of nodes which gives lower bound, least localization error [12]. In this process unknown nodes initiates the localization process and agents in the communication range responds to that process by estimating the locations. All the nodes do not give accurate location due to noise. So selection of nodes which gives accurate locations is important criteria.In this paper CRB is used to select the nodes which gives lower bound, minimum variance of error [13].

We propose to use the CRB on localization error for the selection. Every agent node compares its localization accuracy with all other agents using available global knowledge. This method is known as global-crlb algorithm. This algorithm selects optimal subsets according to the following Equations 1 and 2.

$$
\begin{aligned}
& s(k)=\arg \operatorname{mim} \sigma_{1}^{2}(s) \\
& \sigma_{1}^{2}=\frac{1}{a} \frac{\sum_{i=2}^{M} d_{1, i}^{-2}}{\sum_{i=2}^{M-1} \sum_{j=i+1}^{M}\left(d_{1+i, j}^{2}, d_{i, j}^{2}\right)^{2}} \\
& a=\left(\frac{10 n p}{\sigma_{d B} \ln 10}\right)^{2}
\end{aligned}
$$

$\mathrm{n}_{\mathrm{p}}=$ path loss exponent

$\sigma_{\mathrm{dB}}=$ standard deviation of the received signal strength.

$d_{1 \perp i, j}=$ Distance between anchor and agent.

\subsection{Cooperative Distributive Particle Swarm Optimization (CDPSO)}

PSO is a swarm intelligence algorithm, works on social behavior of birds and a school of fishes [14-15]. It gives a set of solutions called as particles.PSO algorithm complexity is less hence implantation is easy with good convergence [16-17] which is viable for localization of WSN. For improvement in the efficiency and accuracy of PSO-based localization methods are used, distributed PSO algorithm is proposed based on the probabilistic distribution of ranging error. Proposed objective function evaluates the fitness of particles. It attempts to localize more unknown nodes in a high accurate search space [18]. The updated particle's position can be mathematically modelled according the following Equationss 3 and 4.

$$
\begin{aligned}
& \mathrm{V}_{\mathrm{i}}^{\mathrm{k}+1}=\mathrm{w} \mathrm{V}_{\mathrm{i}}^{\mathrm{k}}+\mathrm{c}_{1} \mathrm{r}_{1}\left(\mathrm{p}_{\text {best }}-\mathrm{X}_{\mathrm{i}}^{\mathrm{k}}\right)+\mathrm{c}_{2} \mathrm{r}_{2}\left(\mathrm{~g}_{\text {best }}-\mathrm{X}_{\mathrm{i}}^{\mathrm{k}}\right) \\
& \mathrm{Xi}^{\mathrm{k}+1}=\mathrm{Xi}^{\mathrm{k}}+\mathrm{V}_{\mathrm{i}}^{\mathrm{k}+1}
\end{aligned}
$$

where $\mathrm{v}_{\mathrm{i}}^{\mathrm{k}}, \mathrm{X}_{\mathrm{i}}^{\mathrm{k}}$ are velocity and current position of node $\mathrm{i}$ at iteration $\mathrm{k}, \mathrm{C}_{1}$ and $\mathrm{C}_{2}$ are acceleration constants, $r_{1}$ and $r_{2}$ are random numbers uniformly distributed in $(0,1)$, and $w$ is the inertia weight to control the scope of the search. Usually $w$ is set to linearly decrease with the progression, $\mathrm{p}_{\text {best }}$, $\mathrm{g}_{\text {best }}$ are personal best and global best of the of particles.

Let $(x, y)$ be the coordinates of an unknown node $U$ and $\left(x_{i}, y_{i}\right)$ be the position of its $i_{t h}(i=1,2, \ldots, M)$ neighboring anchor node $A_{i}$ of $U$ and $d_{i}$ be the measured distance between $U$ and $A_{i}$ given by Equations 5 .

$$
\mathrm{F}(\mathrm{x}, \mathrm{y})=\frac{1}{M} \sum_{i=1}^{M} \sqrt{\left(x-x_{i}\right)^{2}+\left(y-y_{i}\right)^{2}-\hat{d}_{i}}
$$

The localization functions $\mathrm{f}$ is defined as all the range-based localization methods with PSO use the objective function as which does not consider the probabilistic distribution of ranging error. If the actual distance $d_{i}$ between $U$ and $A_{i}$ is a normal distribution as given in Equations 6 .

$$
\begin{aligned}
& d_{i} \sim N\left[\hat{d}_{i},\left(\hat{d}_{i} \delta\right)^{2}\right] \\
& \text { wheres }>0
\end{aligned}
$$

The distributions of ranging errors between different nodes are independent, we get $d_{i}$ as given in Equations 7. 


$$
\sum_{i=1}^{M} d_{i} \sim \prod_{i=1}^{M} N\left[\hat{d}_{i},\left(\hat{d}_{i} \delta\right)^{2}\right]
$$

The following Equations 8 with distribution of ranging error is used to improve the location accuracy

$$
\mathrm{f}(\mathrm{x}, \mathrm{y})=\frac{1}{M} \sum_{i=1}^{M} \frac{\left(\sqrt{\left(x-x_{i}\right)^{2}+\left(y-y_{i}\right)^{2}-\hat{d}_{i}}\right)^{2}}{\left(\hat{d}_{i} \delta\right)^{2}}
$$

\section{SIMULATION AND RESULTS ANALYSIS}

\subsection{Simulation setup}

The proposed solution CDPSO with censoring is simulated and tested using MATLAB. 100 agent nodes are randomly placed and 13 anchor nodes with known locations are placed in the area of $500 \mathrm{~m} \times 500 \mathrm{~m}$. The transmission range of every node in one hop is $20 \mathrm{~m}$. standard deviation of measurement is $1 \%$ of measured distance. Figure 2 shows MATLAB-based GUI tool for the calculation of the CRB and localization. $\mathrm{CRB}$ is calculated and displayed on GUI by giving the lower bound on the 2- $\sigma$ ellipse. The threshold parameters $\mathrm{R}_{\mathrm{Tx}}$ is set for the stop limit. The parameters used are $\mathrm{R}_{\mathrm{Tx}}=0.08$ (conservative approach) and the $\mathrm{R}_{\mathrm{Tx}}=0.05$ (aggressive approach). Table 1 shows simulation parameters and values.

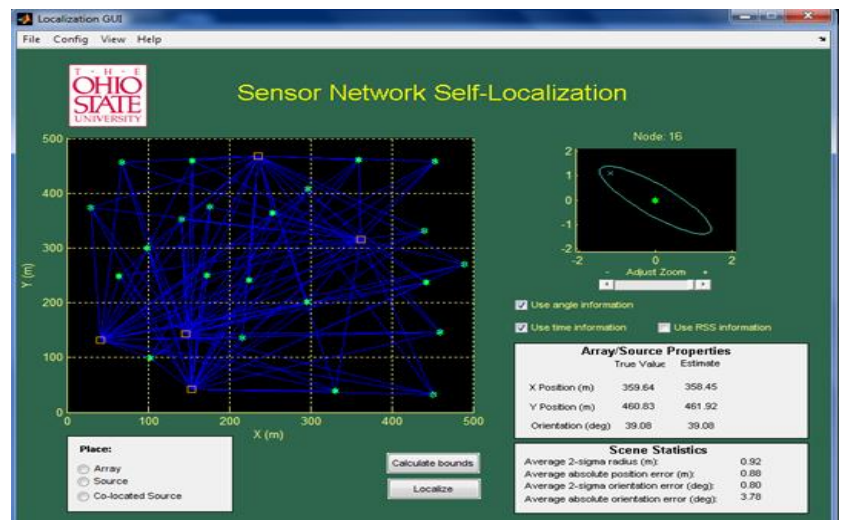

Figure 2. Simulation Setup of Cooperative WSN using Matlab Based GUI Tool

Table 1. Simulation Parameters

\begin{tabular}{lc}
\hline \multicolumn{1}{c}{ Parameter } & Value \\
\hline Simulation area & $500 \mathrm{~m} \mathrm{X} \mathrm{500} \mathrm{m.}$ \\
No.of anchor nodes & 13 \\
No.of unknown nodes & 100 \\
Transmission range $\left(\mathrm{T}_{\mathrm{r}}\right)$ & $20 \mathrm{~m}$ \\
standard deviation $(\boldsymbol{\sigma})$ & $1 \%$ of measured distance \\
Intial energy $\left(\mathrm{E}_{\mathrm{i}}\right)$ & $5 \mathrm{~J}$ \\
Transmission power $(\mathrm{W})\left(\mathrm{P}_{\mathrm{t}}\right)$ & 2 \\
Path loss exponent $(\alpha)$ & 2 \\
Threshold value $\mathrm{R}_{\mathrm{Tx}}($ conservative approach) & 0.08 \\
Threshold value $\mathrm{R}_{\mathrm{Tx}}($ aggressive approach $)$ & 0.05 \\
\hline
\end{tabular}

\subsection{Performance Metrics}

Performane of the proposed algorithm is evaluated by considering different scenarios and performance metrics. The performance metrics for analysis of results are defined below.

a. Cumulative Distribution Function (CDF): CDF is used to measure accuracy of localization system. When two positioning techniques are compared and their accuracies are same then prefer the system with the CDF graph which reaches high probability values faster because its distance error is focused in small values hence $\mathrm{CDF}$ of the distance error is used for measuring the accuracy of a system. 
b. Complexity: Average number of transmissions required to send the data from source to destination in specified intervals of time is known as complexity.

c. Root Mean Square Error (RMSE): Difference between the estimated location value and and actual location value of localization algorithm. Equations 9 gives the formula to find RMSE value.

$$
R M S E=\frac{1}{M} \sqrt{\sum_{k=1}^{m}\left(x_{k}-\hat{x}_{k}\right)^{2}+\left(y_{k}-\hat{y}_{k}\right)^{2}}
$$

\subsection{Results Analysis}

Figure 3 shows the graph plotted for no. of anchors Vs processing time. As the no. of anchor nodes are increasing processing time also increases for the reason that computational complexity, avg. delay also increases. Hence CRB is used to select the optimum reference nodes.



Figure 3. Number of Anchors Vs Processing Time

Figure 4 shows graph plotted for number of anchor nodes Vs location error. Location error is decreased as no. of anchor nodes are increasing. For number of anchor nodes greater than or equal to 4 location errors is minimum and constant.

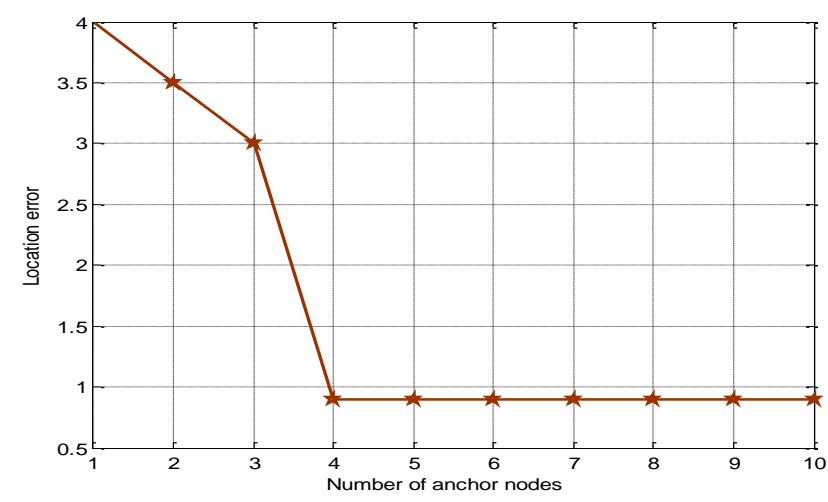

Figure 4. Number of Anchor Nodes Vs Location Error

The performance of proposed method Cooperative Distributed PSO algorithm was tested with different parameters and results are analyzed. Figure 5 represents the graph plotted between position error and $\mathrm{CDF}$ of different censoring schemes in conservative approach $\left(\mathrm{R}_{\mathrm{Tx}}=0.08\right)$. The Distributed PSO algorithm with CRB compared with other algorithms like GPS, LMS, RLS, and PSO in terms of CDF. The CDF of CDPSO with CRB gives better results in comparison with other algorithms. The proposed method discards the transmission of inaccurate location information of nodes, hence selects the nodes which gives accurate results as anchor nodes. In case of PSO the nodes which are in the transmission range of agent nodes 
with inaccurate location information are also used as anchor nodes. Hence the CDF of CDPSO is degraded. In case of RLS, LMS because of less number of nodes which are pre configured participate in the localization process hence CDF is decreased. In case of GPS performance is poor because of NLOS and multipath effects.

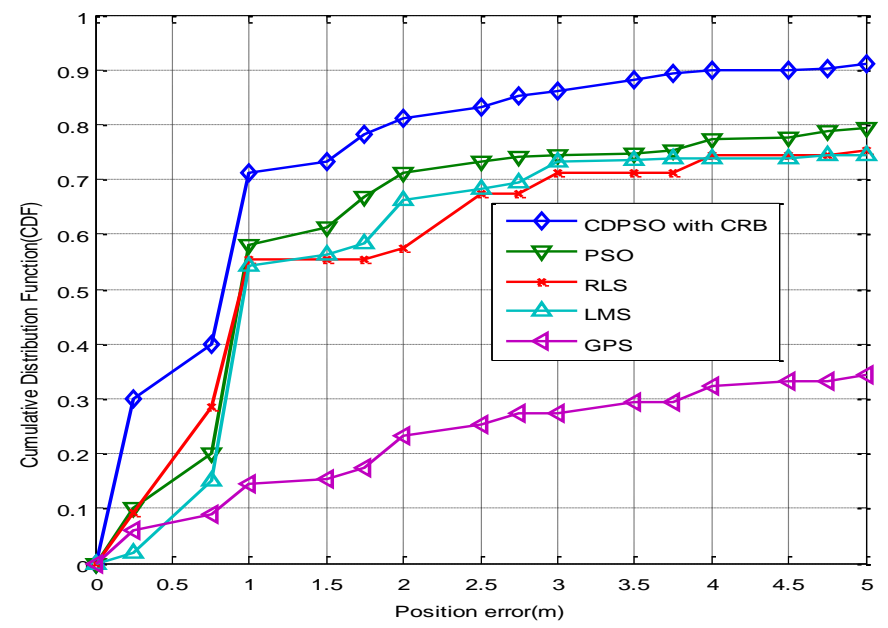

Figure 5. Performance Comparisons of different Censoring Schemes in Conservative Approach

Figure 6 represents the graph plotted between position error and CDF of different schemes in aggressive approach $(\mathrm{RTx}=0.05)$. The CDPSO algorithm with $\mathrm{CRB}$ compared with other algorithms like PSO, RLS, LMS and GPS. The CDF of CDPSO with CRB performs better in comparison with other algorithms because it selects the optimum references with high accuracy, discard location information from inaccurate nodes and localize the nodes using cooperative cooperative distributed PSO. In case of PSO, nodes with inaccurate location information also participate in the localization process. Hence CDF is degraded in comparison with other algorithms. Performance of RLS and LMS is poor because of pre configured nodes only participated in localization process. The performance of GPS is least in comparison with other algorithms due to NLOS, noise and multi path effects.

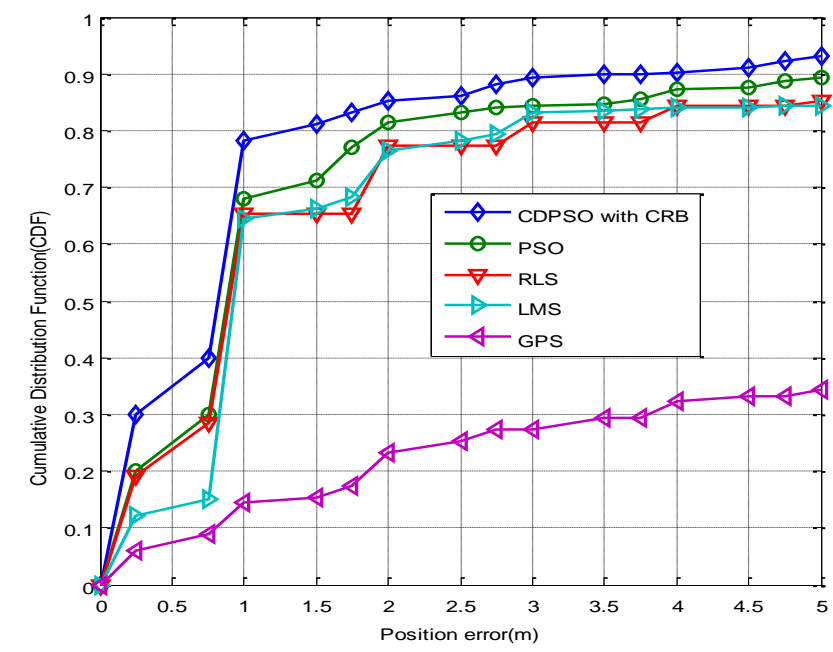

Figure 6. Performance Comparison of different Censoring Schemes in Aggressive Approach

Figure 7 shows graph plotted between number of iterations Vs average number of transmissions per agent. The complexity of CDPSO with CRB is least because of more number of inaccurate nodes are discarded. Hence least number of nodes participates in localization process. Hence complexity is reduced. 
The complexity of PSO is more because of every node in the transmission range participated as reference node in localization process. Hence avg. number of transmissions to localize particular agent node is also increased. In case of GPS signals are affected by noise, multipath effects and computational complexity is more.

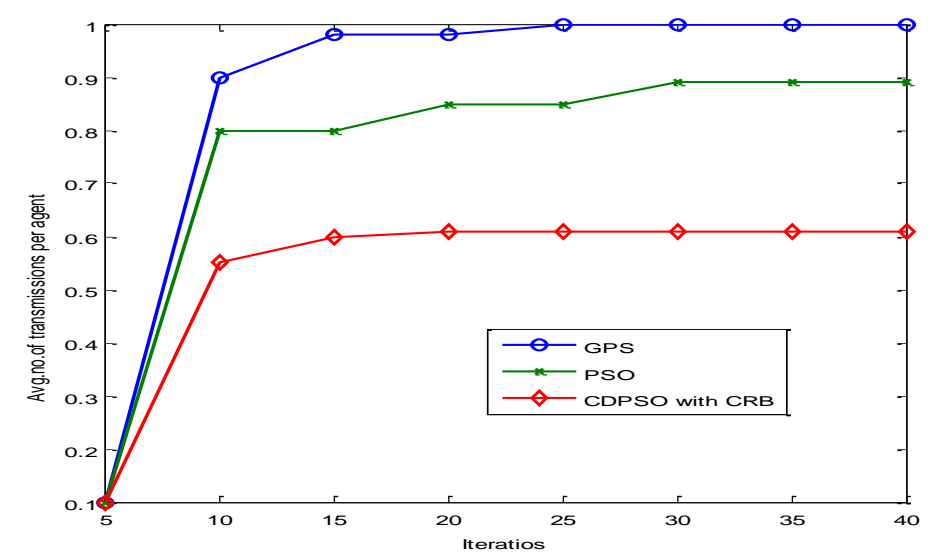

Figure 7. Comparison of different Algorithms for Average Number of Transmissions with Respect to no. of Iterations

Figure 8 represents the graph plotted between number of iterations Vs MSE of location.The CDPSO algorithm with CRB compared with other algorithms PSO, RLS algorithm, LMS algorithm and GPS. Performance of CDPSO algorithm with CRB performs better in comparison with other algorithms because of nodes with accurate information only participates in localization process. In case of PSO inaccurate nodes also participate in localization process, hence the performance is degraded. Performance of LMS and RLS algorithm is poor because of least number of references participated in localization and SNR is low for LMS algorithm. In case of GPS signals are affected by noise and multipath effects hence MSE is more in comparison with other algorithms.

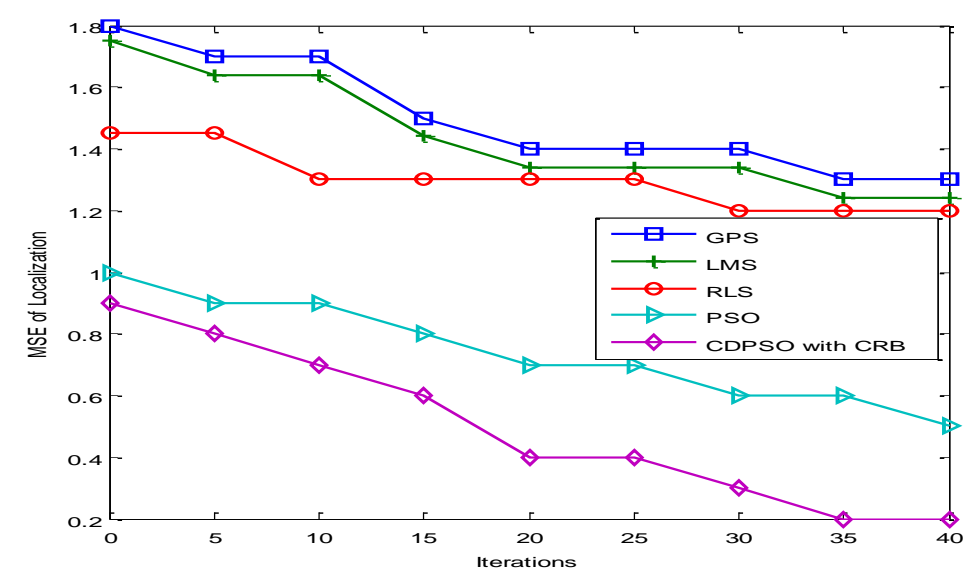

Figure 8. Comparisons of different Algorithms for Mean Square Error of Localization with Respect to no. of Iterations

\section{CONCLUSION}

In this paper CDPSO algorithm with CRB for localization of cooperative WSN is proposed. Performance of the proposed method is studied; simulation tests have been conducted with various conditions. Performance of the proposed method is compared with other existing algorithms like PSO, LMS, 
RLS and GPS algorithm in terms of position accuracy, CDF, complexity and MSE. From the comparison results it is observed that proposed method performs better than other algorithms.

\section{ACKNOWLEDGEMENTS}

The authors would like to acknowledge the financial support of the University Grant commission, India [File No: MRP-6233/15 (SERO/UGC)] to carry out the present research. The authors also acknowledge the Management and Principal of S R EngineeringCollege, Warangal for their continuous support by providing all the necessary facilities. We gratefully thanks to Professors of ECE Department and Research \&Development Cell, JNTU, Anantapur for useful discussion \&suggestions for this research work.

\section{REFERENCES}

[1] D. Chandirasekaran and T. Jayabarathi, "Wireless Sensor Networks Node Localization-A Performance Comparison of Shuffled Frog Leaping and Firefly Algorithm in LabVIEW," TELKOMNIKA Indonesian Journal of Electrical Engineering, vol/issue: 14(3), pp. 516 - 524, 2015.

[2] W. Junsheng, "Design and Application of Iterative Monte Carlo Localization for Mobile Wireless Sensor Networks Based on MCL," TELKOMNIKA Indonesian Journal of Electrical Engineering, vol/issue: 12(3), pp. 2079 -2087, 2014.

[3] Sivieri A., et al., "Building Internet of Things software with ELIoT," Computer Communications, 2016.

[4] Lv T., et al., "Space-time hierarchical-graph based cooperative localization in wireless sensor networks," Signal Processing, IEEE Transactions, vol/issue: 64(2), pp. 322-34, 2016.

[5] Ramasamy D., et al., "Compressive parameter estimation in AWGN," Signal Processing, IEEE Transactions, vol/issue: 62(8), pp. 2012-27, 2014.

[6] Yang G., et al., "Throughput optimization for massive MIMO systems powered by wireless energy transfer," Selected Areas in Communications, IEEE Journal, vol/issue: 33(8), pp. 1640-50, 2015.

[7] A. Conti, et al., "Network experimentation for cooperative localization," IEEE J. Sel. Areas Commun, vol/issue: 30(2), pp. 467-475, 2012.

[8] E. Garcia, et al., "On the trade-off between accuracy and delay in cooperative UWB navigation," in proc. Int. conf. Wireless Communications and Networking, Shanghai, pp. 1603-1608, 2013.

[9] Garcia G. E., et al., "On the trade-off between accuracy and delay in cooperative UWB localization: Performance bounds and scaling laws," Wireless Communications, IEEE Transactions, vol/issue: 13(8), pp. 4574-85, 2014.

[10] Das K. and Wymeersch H., "Censoring for bayesian cooperative positioning in dense wireless networks," Selected Areas in Communications, IEEE Journal, vol/issue: 30(9), pp. 1835-42, 2012

[11] Savic V. and Zazo S., "Cooperative localization in mobile networks using nonparametric variants of belief propagation," Ad Hoc Networks, vol/issue: 11(1), pp. 138-50, 2013.

[12] Lv T., et al., "Space-time hierarchical-graph based cooperative localization in wireless sensor networks," Signal Processing, IEEE Transactions, vol/issue: 64(2), pp. 322-34, 2016.

[13] Gribben J. and Boukerche A., "Location error estimation in wireless ad hoc networks," Ad Hoc Networks, vol. 13, pp. 504-15, 2014.

[14] Ma'sum M. A., et al., "Automatic fetal head approximation using Particle Swarm Optimization based Gaussian Elliptical Path," in 2015 International Symposium on Micro-NanoMechatronics and Human Science (MHS), pp. 16, 2015.

[15] Miraswan K. J. and Maulidevi N. U., "Particle swarm optimization and fuzzy logic control in gas leakage detector mobile robot," in 2015 International Conference on Automation, Cognitive Science, Optics, Micro ElectroMechanical System, and Information Technology (ICACOMIT), pp. 150-155, 2015.

[16] Esmin A. A., et al., "A review on particle swarm optimization algorithm and its variants to clustering highdimensional data," Artificial Intelligence Review, vol/issue: 44(1), pp. 23-45, 2015.

[17] Liu J., et al., "A Cooperative Evolution for QoS-driven IoT Service Composition," Automatika-Journal for Control, Measurement, Electronics, Computing and Communications, vol/issue: 54(4), 2013

[18] Yao Y. and Jiang N., "Distributed wireless sensor network localization based on weighted search," Computer Networks, vol. 86, pp. 57-75, 2015.

\section{BIOGRAPHIES OF AUTHORS}



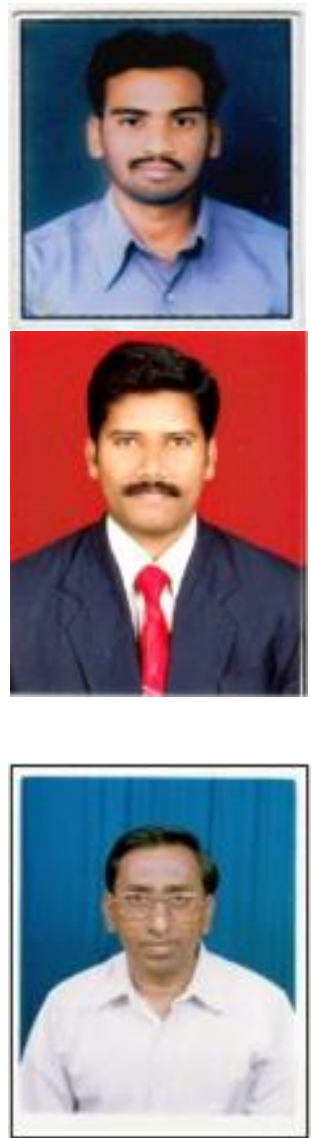

Ravichander Janapati received B.Tech degree in Electronics and Communication Engineering from JNTU Hyderabad. He received his M.Tech degree in Digital Electronics and Communication Systems from JNTU Anantapur. Now, he is Research Scholar of Jawaharlal Nehru Technological University, Anantapur in the Area of Adhoc Wireless Sensor Networks and signal processing and Life Time Member of IETE. His Reaserch Papers published in Five International Journals and Six International Conferences.Now $\mathrm{He}$ is working as Senior Assistant Professor in SR Engineering college,Warangal,India.

Ch. Balaswamy received the B.E degree in Electronics and Communication Engineering from S.R.K.R. Engineering College, Bhimavaram in 1998. He received the M.Tech degree in Electronics and Communication Engineering from Malnad College of Engineering, Hassan, India in 2001. He worked as assistant professor in various engineering colleges in Andhra Pradesh, India. He worked as associate professor in LakireddyBalireddy College of Engineering, Krishna district, India from August 2005 to October 2006. he Completed PhD from Jawaharlal Nehru Technological University, Anantapur.His areaof Interest is Mobile Adhoc Networks, Micro Processors \& Controllers and Embedded System. He has published eight International Journals and Six Researchpapers in National and International Conferences. Now He is Life Time Member of IETE, Professor \& HOD Of ECE, QIS College of Engg \& Tech

K. Soundararajan received the B.E degree in Electronics and Communications from S.V.U, Tirupati and the M.Tech degree in Instrumentation from J.N.T.U, Kakinada. He received Ph.D from Indian Institute of Technology, Roorkee. He is having 26 years of teaching experience. He got the best teacher award for the year 2005, President of India Award in Bharat Scouts \& Guides in 1968, Best Paper Award in 1990-91 from Institution of Engineers (India) for best technical paper published in Journal and the Best Teacher Award in 2006 by the State Government of Andhra Pradesh, India. As a Researcher, he has successfully guided Nine $\mathrm{Ph}$. Dsin addition, to one in progress resulting in 27 International Jnl.s / Conf.Proc. , 32 national Jnls. / Conf. Proc. and 11 National seminars. 